\title{
Is the Prefrontal Cortex Especially Enlarged in the Human Brain? Allometric Relations and Remapping Factors
}

\author{
Richard E. Passingham ${ }^{a} \quad$ Jeroen B. Smaers ${ }^{b}$ \\ ${ }^{a}$ Department of Experimental Psychology, University of Oxford, Oxford, UK; ${ }^{\text {b }}$ Department of Anthropology, \\ Stony Brook University, New York, N.Y., USA
}

\author{
Key Words \\ Prefrontal cortex $\cdot$ Cytoarchitectonic criteria · Human . \\ Great apes · Monkey
}

\begin{abstract}
There has been no agreement as to whether the prefrontal cortex is especially enlarged in the human brain. To answer this question, we analyzed the only two datasets that provide information on total prefrontal cortex volume based on cytoarchitectonic criteria. One delineated the prefrontal cortex proper on the basis of cytoarchitectonic criteria; the other used a proxy of the prefrontal cortex based on a cytoarchitectonic delineation of the frontal lobe. To investigate whether all cortical association areas, including the prefrontal cortex, are enlarged in the human brain, we scaled the different areas to a common reference, the primary visual cortex. To investigate whether the prefrontal cortex is more enlarged than other association areas, we scaled it relative to its inputs from and outputs to other nonprimary areas. We carried out separate regression analyses using different data samples as a predictive baseline group: data for monkeys alone informs us on whether great apes are different from monkeys; data for all non-human anthropoids, including great apes, informs us on whether humans are different from all other primates. The analyses show that the value for the human prefrontal cortex is greater than expected, and that this is true even
\end{abstract}

when data for the great apes are included in the analysis. They also show that the chimpanzee prefrontal cortex is greater than expected for a monkey with a similar sized cortex. We discuss possible functional consequences.

(c) 2014 S. Karger AG, Basel

\section{Introduction}

There has been no agreement as to whether either the frontal lobes [Semendeferi et al., 2002; Barton and Venditti, 2013] or the prefrontal cortex in particular [Deacon, 1997; Holloway, 2002] are especially enlarged in the human brain. One way to find out is to fit a regression line through the values for non-human primates and so calculate the size expected for a hypothetical primate with as large a neocortex [Passingham, 1973]. The difference between the predicted and actual value can then be assessed to see if it is statistically significant.

There are three reasons why the issue is still not settled. The first is the poverty of the datasets. The second is uncertainty as to which non-human primates form the most appropriate comparison. The third is lack of agreement on the part of the brain with which to compare the prefrontal cortex.

The reason for the lack of extensive data is that the prefrontal cortex should be defined on the basis of cytoarchi-

\section{KARGER}

E-Mail karger@karger.com

www.karger.com/bbe (c) 2014 S. Karger AG, Basel

0006-8977/14/0842-0156\$39.50/0
Richard E. Passingham

Department of Experimental Psychology

University of Oxford, South Parks Road

Oxford OX1 3UD (UK)

E-Mail dick.passingham@ @sy.ox.ac.uk 
tecture [Passingham and Wise, 2012]. It is distinct from the neighboring premotor and motor cortices in having a distinct fourth granular layer [Mackey and Petrides, 2010]. Thus, a practiced eye is needed to distinguish the layers under a light microscope, and so the task of comparing the prefrontal cortex in many species is very laborious indeed. This has meant that there are only three datasets based on a cytoarchitectonic analysis.

The first comes from Brodmann [1913] and is for the granular prefrontal cortex as a whole. The data are tabulated in Elston [2007]. Unfortunately, it is a limitation that values are given for only one individual for each of the non-human primate species. This matters because there can be considerable variation among individuals [Semendeferi et al., 2002]. The sample for this dataset includes human, chimpanzee, gibbon and 7 monkeys.

The second dataset comes from Semendeferi et al. [2001]. This is for the frontopolar cortex (area 10) alone. It also provides values for apes, but not monkeys. However, it too gives values for only one individual for each species.

The third dataset comes from Smaers et al. [2011]. They defined the frontal lobe based on cytoarchitectonic criteria as the border between areas 3 and 4 . They avoided defining the posterior border of the prefrontal cortex by dividing the frontal lobe into 20 sections and then taking volume 5 , reading back from the frontal pole, as being certain to lie within the prefrontal cortex. They then demonstrated that the results were the same if they considered volumes $4-10$. The dataset provides information on white and gray matter, and does so separately for each hemisphere. It covers 10 humans, 4 great apes, gibbon and 13 monkeys. The data for non-human primates provide values for one individual for each species.

Not only are the data limited, but there is also the further problem that it is not clear which primates form the most appropriate comparison. One could assess the human prefrontal cortex against the values for all anthropoids, that is monkeys and apes, or for the great apes alone. Our nearest relatives are great apes. We shared a common ancestor with monkeys 23 or more million years ago, but with chimpanzees between 5 and 7 million years ago [Kumar et al., 2005]. However, while it is possible to gather data on the great apes by scanning them with MRI [Rilling and Seligman, 2002], it is not yet possible to delineate cytoarchitectonic areas on the basis of MRI scans, though there is hope for the future that areas might be delineated on the basis of their myelin content [Glasser et al., 2013].

We suggest that the most appropriate comparison group depends on the question to answer. When investi-

Is the Prefrontal Cortex Especially

Enlarged in the Human Brain? gating whether humans follow primate predictions, the best baseline comparison is that with non-human primates. When investigating whether great apes follow primate predictions, the best comparison is that with monkeys [Smaers, 2013]. We have included the gibbon in the regression with the monkeys in the current study because the values for the gibbon are well within the range for monkeys.

The final issue is the most critical. It has been usual to compare the prefrontal cortex either with the rest of the neocortex [Passingham, 1973] or with the rest of the brain [Semendeferi et al., 2001]. While this will tell us whether the prefrontal cortex is enlarged, it is not sufficient to tell us whether it is the only area that is enlarged. It could be that some other area, for example the parietal association cortex, is also enlarged. We therefore make two comparisons. First, we compare each area with a common reference point, the primary visual cortex. Second, we compare the association areas with each other.

In the present paper we have chosen to use the size of the primary visual or striate cortex as a common reference. It would have been better to use the size of all the primary sensory areas together as they provide the cortical inputs to the nonprimary areas. These areas have a characteristic cytoarchitecture in that they have a very well-developed fourth layer with many small granule cells. This is true 'koniocortex'. Though we have measures for koniocortex, it is only for five haplorhines including humans [Shariff, 1953].

It could be objected that if the values for striate cortex are plotted against brain size, the human striate cortex is smaller than expected for a primate with a brain as large [de Sousa et al., 2010]. However, there are two possible interpretations. The first is that the striate cortex is indeed too small. The second is that it is the rest of the brain that is expanded. The only way to find out is to assess the striate cortex against some other reference point. This can be done by plotting the striate cortex either against body size or against the size of the lateral geniculate nucleus. The human striate cortex is as predicted for a primate of the same body size [Passingham, 1973] and it is also as predicted for a primate with the same size of lateral geniculate [Bush and Allman, 2004].

This is also true of all the primary sensory areas. The human value for koniocortex as a whole is as predicted for a primate of the same body size [Passingham, 1975]. Unfortunately, we have no measure for motor cortex on its own, but the size of the human pyramidal tract is as expected for a primate of our size [Passingham, 1981]. We may conclude that in the human brain the primary 
sensory and motor areas scale with our body weight. The association cortex is the 'eulaminate cortex' [Bailey and von Bonin, 1951], and it is the eulaminate cortex that is much more extensive than expected for a primate of our body size [Passingham, 1975].

The advantage of using the primary sensory cortex as a reference point is that there is a theoretical justification for doing so. The body surface, including the retina, is mapped onto primary sensory areas [Kaas, 1997]. In turn, the representations in these areas are mapped onto secondary areas and then onto association areas. So, for example, in the ventral visual system V1 projects to the secondary areas $\mathrm{V} 2$ and $\mathrm{V} 4$, and V4 projects in turn to the inferotemporal cortex [van Essen, 2005]. Within this stream there is a hierarchical analysis such that the optimal stimulus becomes more complex [Desimone and Schein, 1987] and more object like [Tanaka, 1993]. Thus, the processing capacity of the ventral visual system can be assessed by relating the amount of tissue in higher areas that is available for analyzing the sensory information that is represented in the primary visual cortex. We call this the 'remapping factor' by analogy with the cortical magnification factor in the visual cortex [Cowey and Ellis, 1969].

\section{Methods}

\section{Samples}

We analyzed the only two datasets that provide information on total prefrontal cortex volume based on cytoarchitectonic criteria. Brodmann [1913] used cytoarchitectonic criteria only and provided information on the granular frontal cortex, agranular cortex, total neocortex and striate cortex $\left(\mathrm{mm}^{2}\right)$. Unfortunately, Brodmann did not provide separate values for the motor cortex and the premotor areas. The latter comprise the premotor, supplementary motor, presupplementary motor and cingulate motor areas. In this paper we use the term 'frontal motor areas' to refer to the combination of these areas. The data that we take from Brodmann are for 6 of the 7 monkeys, the gibbon, the chimpanzee and 1 human. We have not used the data for the guenon in the Brodmann dataset because he does not give a striate cortex value for this animal.

Smaers et al. [2011] provide a more complete comparative sample. As already mentioned, it includes values for 13 monkeys, gibbon, orangutan, gorilla, bonobo, chimpanzee and human. This dataset relies on the cytoarchitectonic delineation of the frontal lobe as the border between area 3 and 4, using six cytoarchitectonic criteria: cell shape (absence vs. presence of pyramidal and elongated Betz cells); cell density (high vs. low); cell size (small vs. large); granular layer IV (present vs. absent); border between white and gray matter (sharp vs. diffuse), and lamination (sharp vs. diffuse) [for more information, see Smaers et al., 2010]. As explained in the introduction, the sections are then measured at intervals from the frontal pole [Smaers et al., 2010, 2012, 2013]. The supplementary material gives an example of this delineation procedure and the cytoarchitectonic delineation of the border between area 3 and 4 (see suppl. material; see www.karger.com/doi/10.1159/000365183 for all online suppl. material). The figures presented in the main text relate to analyses using the 5 th section interval. The reason why the 5 th section interval is displayed here is because this section interval is certain to include the prefrontal cortex volume and lies prior to an observed shift at the 8th section interval in white-to-gray matter ratio in apes [Smaers et al., 2011]. Importantly, scaling results for all other section intervals (4th, 6th, 7th, 8th, 9th and 10th) give similar results (see suppl. material). The individuals used in the Smaers dataset come from a collection of serial histological sections housed at the Vogt Institute for Brain Research [Zilles et al., 2011].

To subdivide the neocortex into as many relevant areas as possible, we complemented the Brodmann and Smaers datasets with additional information from the literature. Using Brodmann's own data on total neocortex, striate, prefrontal and premotor cortex plus motor cortex, we calculated the nonfrontal cortex, and by subtracting the values for striate cortex we estimated the areas for the temporal plus parietal plus prestriate cortex.

To further subdivide this area we used information on the temporal and superior temporal cortex from another dataset [Rilling and Seligman, 2002]. From this we were able to calculate values for the inferotemporal cortex. In the human and chimpanzee brain this measure includes the middle and inferior temporal gyrus. We then used values for striate cortex given by Brodmann where available for the species in the Rilling dataset. To arrive at an estimate of the parietal cortex we used the data on the nonfrontal cortex from Brodmann and the data on the temporal cortex from Rilling and Seligman. By subtracting the temporal from the nonfrontal cortex and then subtracting the striate cortex we were able to arrive at an estimate of the parietal plus prestriate cortex. We acknowledge that these analyses are approximate as the individuals for any species in the Brodmann and Rilling datasets are not the same and there is variation among individuals.

To complement the Smaers dataset we used information on frontal and neocortical gray matter from Smaers et al. [2010] to calculate the nonfrontal cortex; that is temporal plus parietal plus prestriate plus striate. We then used volumes of striate gray matter from Frahm et al. [1984] and de Sousa et al. [2010] to calculate the nonfrontal minus striate cortex. The data used for these calculations come from the same individuals, all from the collections in the Vogt institute [Zilles et al., 2011].

\section{Statistical Methods}

We use phylogenetic generalized least squares (PGLS) analysis with a likelihood-fitted lambda model [Orme et al., 2012] to fit a regression line to the data. The reason for this is that, if standard linear regression is used, the slope drawn through closely related species may appear to be shallower than the slopes drawn through distantly related species [Kelly and Price, 2004]. To correct for this, the statistical method must take into account the error structure expected given the phylogenetic relatedness of the species studied. PGLS incorporates this error structure by weighting observations according to a variance-covariance matrix of relative phylogenetic distance between species; this is independent of the depth of the inferred root of the tree. PGLS is thus a standard regression in which the estimates of the scaling coefficients are calculated based on weighted observations [Rohlf, 2001].

Confidence intervals of PGLS scaling coefficients should be calculated using the standard procedures, that is scaling coefficient \pm 1.96 SE for the population, with the $t$ score (' 1.96 ') adjusted for 

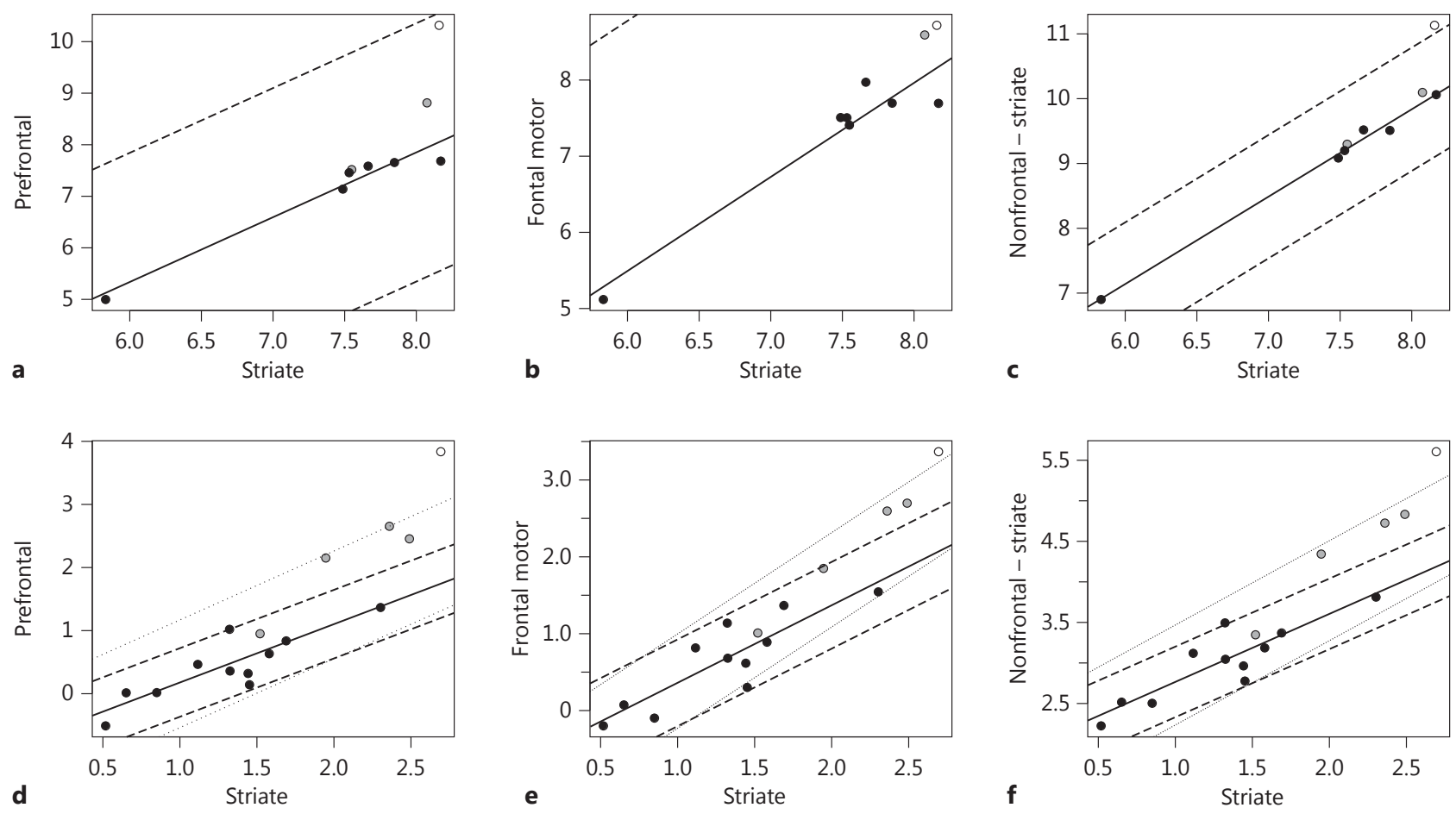

Fig. 1. a-c The Brodmann dataset. The solid line shows the regression through the data for monkeys and the gibbon, and the hatched lines show the $95 \%$ confidence limits. The values for chimpanzee and gibbon are shown in gray and the value for the human brain by an open circle. The data are in millimeters squared $\left(\mathrm{mm}^{2}\right)$ and are plotted on a log-log scale. $\mathbf{d - f}$ The Smaers dataset. The conven-

the respective degrees of freedom [Rao and Toutenburg, 1999]. Because our primary focus is to test whether particular species indicate a higher than expected value for particular brain areas, we focus our analysis on the confidence interval of the intercept only. This procedure adequately describes the occurrence of grade shifts and has become commonplace in similar analyses [Barton and Harvey, 2000; Barton and Venditti, 2013].

It is a limitation of the present study that, with so few values through which to plot regressions, the $95 \%$ confidence limits are very wide. As a consequence, even when the absolute difference between the predicted and obtained values is large, it is not always statistically significant. This limitation reflects the uncertainty of model predictions based on small sample size and raises the question as to whether, when dealing with such small samples, statistical cut-offs mask potentially biologically meaningful changes [Sherwood and Smaers, 2013]. In the text we therefore give the ratios of observed to predicted values and also state whether the differences are statistically significant. Where the differences are large but not significant they should be treated as hypotheses that await testing with more adequate samples.

Is the Prefrontal Cortex Especially

Enlarged in the Human Brain? tions are the same, except that the data for all apes (4 great apes as well as the gibbon) are shown in gray. In addition, the dotted lines show the $95 \%$ confidence limits for a regression through the data for all the non-human primates, that is including the great apes. The data are in millimeters cubed $\left(\mathrm{mm}^{3}\right)$ and are plotted on a loglog scale.

\section{Results}

We distinguish three issues. The first is which, if any, areas within the association cortex are enlarged in the human brain, and to tackle this we used the striate cortex as a reference point. The second issue is whether the prefrontal cortex is especially enlarged, and to find out we compared it with other neocortical areas. The final issue is what the consequences of such an enlargement are in terms of processing capacity, and we assessed this in terms of the remapping factors.

\section{Predictions on the Basis of Primary Visual Cortex}

We first fitted regression lines through the data for different brain areas on a log-log scale, using the striate cortex as a common reference point. Figure 1 shows the results for the Brodmann (fig. 1a-c) and Smaers (fig. 1d-f) 
Table 1. The slopes for the regressions

\begin{tabular}{|c|c|c|c|}
\hline \multirow[t]{2}{*}{ Slope } & \multicolumn{2}{|l|}{ MonkeyG } & \multirow{2}{*}{$\frac{\mathrm{NH}}{\text { Smaers }}$} \\
\hline & Brodmann & Smaers & \\
\hline Prefrontal vs. striate & 1.26 & 0.92 & 1.09 \\
\hline Frontal motor vs. striate & 1.24 & 1.01 & 1.32 \\
\hline Nonfrontal - striate vs. striate & $1.35^{*}$ & 0.84 & 1.04 \\
\hline Prefrontal vs. frontal motor & 0.98 & 0.89 & 1.07 \\
\hline Prefrontal vs. nonfrontal - striate & 0.93 & 1.12 & 1.18 \\
\hline
\end{tabular}

The asterisk indicates that the slope is significantly different from $1(\mathrm{p}<0.05)$. MonkeyG = Monkey plus gibbon; $\mathrm{NH}=$ the 4 great apes.

datasets. For each dataset the figure shows plots for the prefrontal cortex (fig. 1a, d), the frontal motor areas (fig. $1 \mathrm{~b}, \mathrm{e}$ ), and nonfrontal minus striate cortex relative to the striate cortex (fig. 1c, f). Values for the Brodmann dataset refer to cortical surface area; values for the Smaers dataset to cortical gray matter volume. The values for the slopes of the regression lines are given in table 1.

Given the unreliability of fitting a regression line through the four values for the great apes, the line through all the non-human anthropoids in the Smaers dataset provides the most rigorous test of whether the human values are or are not as predicted when the values for great apes are taken into account. The regression line through monkeys plus gibbon provides a test of whether great apes are or are not as predicted considering other primates.

The ratio of the observed to predicted values are given in table 2. The first analysis (top row of table 2) compares the prefrontal cortex with the value predicted on the basis of the striate cortex. For the Smaers dataset the human value for the prefrontal cortex is greater than predicted $(p<0.05)$ on the basis of regression through the values for monkeys and the gibbon, and for the regression through the values for all non-human primates (fig. 1d). The values for the great apes also differ significantly from those predicted for monkeys and the gibbon (fig. 1d; table 2). Because the Smaers dataset is comprised of cumulative volumes along the frontal pole (see online suppl. material), we carried out separate analyses of each of the volumes from section intervals $4-10$. The results are relatively stable over those volumes; they are presented in the online supplementary material.

For the Brodmann dataset, the ratios of observed to predicted values for the human and chimpanzee are comparable to those of the Smaers dataset, although both fail to reach significance (fig. 1a). The human value in the Brodmann dataset, however, nears the 0.05 significance level ( $\mathrm{p}<0.07$; fig. $1 \mathrm{a}$ ), and would be significant if a onetailed analysis were adopted.

The next analyses (rows two and three of table 2) present the data for frontal motor areas and for the nonfrontal minus striate cortex. For the Smaers dataset, both these areas are significantly greater than predicted in the human brain. This is true whether the comparison is with monkeys and the gibbon alone or with the addition of the great apes. These areas are also significantly greater than predicted in great apes when considered in reference to monkeys and the gibbon. It is unfortunate that we do not have data for the premotor areas independently of the motor cortex so as to assess whether the finding for the frontal motor areas reflects an expansion of the nonprimary motor areas.

For the Brodmann dataset, the human nonfrontal cortex is significantly greater than predicted, though the value for the motor areas does not reach significance. The difference between the results for the two datasets is probably due to the difference in the slopes of the regression lines. The slopes are shown in table 1. The slopes are isometric in the Smaers dataset, that is, the slopes do not differ significantly from 1 . However, the slopes are hypermetric in the Brodmann dataset. The reason is that the slope in the Brodmann dataset is influenced by the inclusion of the marmoset as an outlier. If the value for the marmoset is excluded from the analysis of the Brodmann data, the slopes are no longer hypermetric, thus aligning with results from the Smaers dataset.

\section{Human Prefrontal Cortex Compared with Other \\ Areas}

The previous analyses showed that the prefrontal cortex is larger than predicted, but that it is not the only area that is larger than predicted in the human brain. To explore whether the prefrontal cortex is more enlarged than other areas, we also regressed the prefrontal cortex relative to other neocortical areas (fig. 2). In these analyses, the comparison of the human residual value with the values for other areas informs us on whether the human prefrontal cortex is especially enlarged. The values for the slopes are given in table 1.

For both datasets the human prefrontal cortex is greater than would be predicted for the frontal motor areas on the basis of the monkeys and gibbon (table 2; fig. 2a, c). Furthermore, for the Smaers dataset the human prefrontal cortex is also greater than predicted even when the great apes are added. 
Table 2. The ratio of observed to predicted values

\begin{tabular}{|c|c|c|c|c|c|}
\hline \multirow[t]{2}{*}{ Observed/predicted } & \multicolumn{2}{|c|}{ Chimpanzee } & \multicolumn{3}{|l|}{ Human } \\
\hline & \multicolumn{2}{|l|}{ MonkeyG } & \multicolumn{2}{|l|}{ MonkeyG } & $\frac{\mathrm{NH}}{\text { Smaers }}$ \\
\hline Prefrontal vs. striate & 2.38 & $3.38^{*}$ & 9.64 & $8.10^{*}$ & $5.31^{*}$ \\
\hline Frontal motor vs. striate & 1.71 & $2.37^{*}$ & 1.75 & $3.66^{*}$ & $2.12^{*}$ \\
\hline Prefrontal vs. nonfrontal - striate & 2.05 & 1.34 & 3.51 & $1.63^{*}$ & $1.36^{*}$ \\
\hline
\end{tabular}

The entries indicate how many times larger the observed values are compared with the predicted values. An asterisk indicates that the value lies outside the $95 \%$ prediction interval for the corresponding regression. MonkeyG $=$ Monkey plus gibbon; $\mathrm{NH}=\mathrm{monkeys}$ plus the 4 great apes.

Fig. 2. a-d The conventions are as for fig-
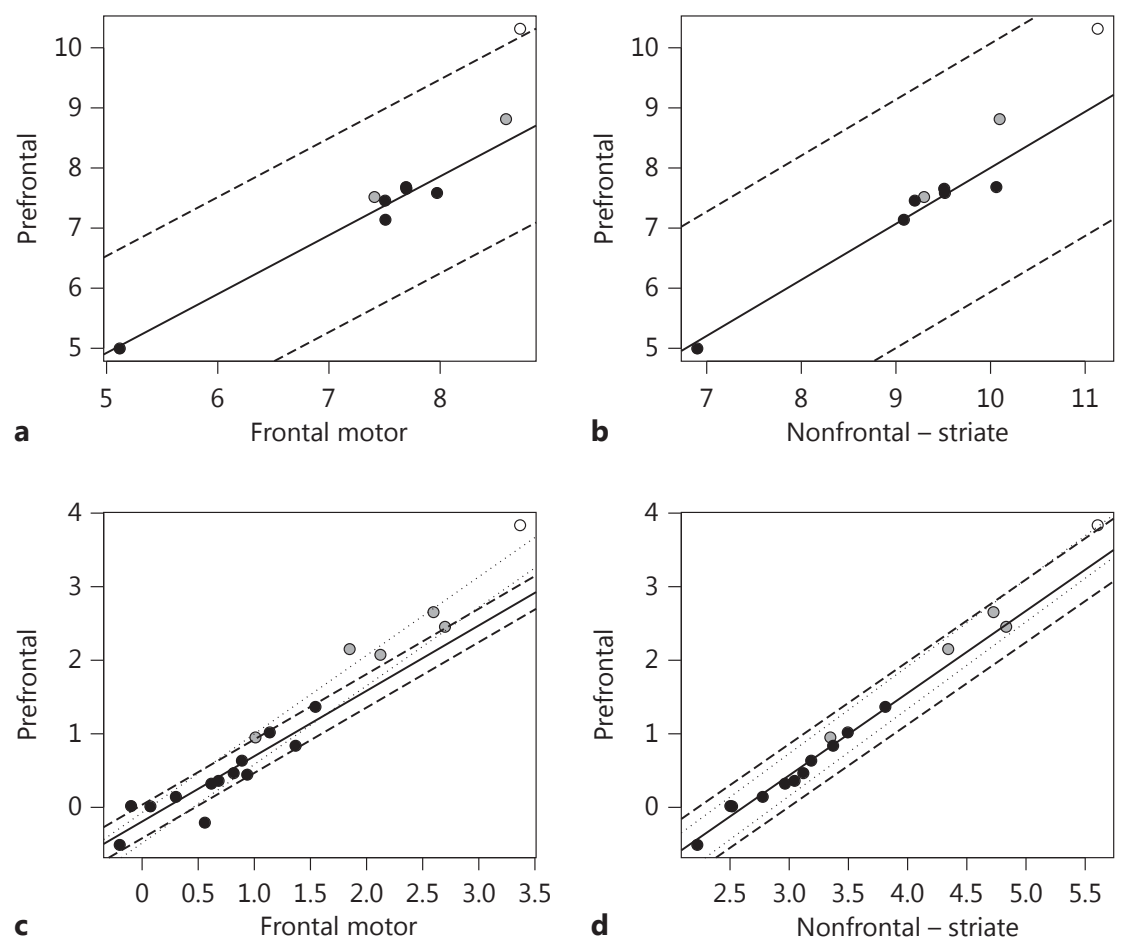
ure 1.

Figure $2 \mathrm{~d}$ and the last row in table 2 show that for the Smaers dataset the human prefrontal cortex is also greater than predicted for the nonfrontal minus striate cortex. A more detailed analysis of the prefrontal to nonfrontal minus striate cortex regression across section intervals for the Smaers dataset (see online suppl. material) reveals that the human value is larger than predicted at a 0.05 significance level for the most anterior section intervals of the prefrontal cortex, with more posterior section intervals nearing significance levels between 0.05 and 0.10 (see online suppl. material). Ratios of observed to predicted values for these analyses are consistently between 1.20 and 1.56 across intervals. 
Table 3. Remapping factors for the human, chimpanzee and macaque monkey brain

\begin{tabular}{lcllll}
\hline & Human & Chimp & Macaque & $\begin{array}{l}\text { Human vs. } \\
\text { chimp }\end{array}$ & $\begin{array}{l}\text { Chimp vs. } \\
\text { macaque }\end{array}$ \\
\hline Prefrontal/striate & 8.86 & 2.09 & 0.92 & 4.14 & 2.27 \\
Frontal motor/striate & 1.75 & 1.68 & 0.97 & 1.04 & 1.73 \\
Nonfrontal - striate/striate & 19.55 & 7.55 & 5.30 & 2.59 & 1.42 \\
Parietal + prestriate/striate & 13.96 & 5.44 & 3.71 & 2.57 & 1.47 \\
Inferotemporal/striate & 5.38 & 1.48 & 1.07 & 3.63 & 1.38 \\
Prefrontal/frontal motor & 4.94 & 1.25 & 0.95 & 3.95 & 1.31 \\
Prefrontal/nonfrontal - striate & 0.44 & 0.28 & 0.17 & 1.57 & 1.65 \\
Prefrontal/inferotemporal & 2.15 & 1.41 & 0.87 & 1.52 & 1.62 \\
\hline
\end{tabular}

The two columns on the right compare the remapping factors for the human and chimpanzee brain, and for the chimpanzee and macaque monkey brain.

The human residual of the prefrontal to nonfrontal minus striate cortex did not reach significance for the Brodmann data (fig. $2 \mathrm{~b} ; \mathrm{p}<0.18$ ). However, the ratio of observed to predicted value is 3.51 for the human and 2.05 for the chimpanzee (table 2).

\section{Remapping Factors}

The allometric results (table 2) show the difference between the values for the human brain and those predicted for a primate matched either for the size of striate cortex (fig. 1) or for that of other areas (fig. 2). Analyses of this sort can be considered as concerning developmental neuroanatomy. They tell us whether the changes in the pattern of development that would produce the neocortex of primate $\mathrm{A}$ rather than the neocortex of primate $\mathrm{B}$ could also produce the human neocortex. In other words, if the human value is as predicted, this suggests that the same developmental pattern is followed. These analyses concern how the human brain evolved.

The remapping factors (table 3 ) show the difference between the amount of tissue in particular neocortical areas compared with their inputs or outputs. Analyses of this sort can be considered as concerning cognitive neuroscience. The assumption is that the greater the amount of tissue in relation to the inputs or outputs, the greater the processing capacity. We show the remapping factors for the macaque monkey, chimpanzee and human neocortex, but intend no suggestion that they form an 'evolutionary series'. The table gives the values for the Brodmann dataset. We present data for only three species for simplicity of presentation.

The analyses of remapping factors concern the effects of any changes in evolution. Irrespective of whether the human brain follows or does not follow a similar devel- opmental course to that of other anthropoids, it is a separate question whether any changes had consequences for processing capacity.

When the remapping factors are assessed in relation to the primary visual input, the values for the nonprimary areas are between 2.57 and 4.14 times bigger in the human compared with the chimpanzee brain. When the remapping factor for the prefrontal cortex is assessed in relation to its parietal and temporal inputs, the value for the human brain is 1.57 times that for the chimpanzee brain. Unfortunately, our measure of the parietal and temporal cortex includes the primary areas SI, SII and the auditory cortex. However, the measure of inferotemporal cortex does not, consisting of the association cortex alone. The remapping factor for the human prefrontal cortex as assessed in relation to the inferotemporal cortex is 1.52 times that for the chimpanzee. This does not differ appreciably from the value as assessed in relation to the nonfrontal minus striate cortex.

When the remapping factor for the prefrontal cortex is assessed in relation to its outputs, the value for the human brain is 3.95 times that for the chimpanzee brain. However, motor cortex is included in the frontal motor areas, and this scales with body size. It is unfortunate that we do not have data for the premotor areas alone. It is likely that the remapping factor of prefrontal to premotor areas would be smaller.

\section{Discussion}

This paper has considered whether the prefrontal cortex is especially enlarged in the human brain. To answer this question we first compared the size of the prefrontal 
cortex relative to the size of the striate cortex. The justification is that the striate cortex provides an input, and can therefore serve as a common reference point across species. To assess whether the prefrontal cortex is especially enlarged we compared the prefrontal cortex with the size of other association areas.

Is the Human Prefrontal Cortex Larger than Expected?

The results indicate that in both humans and great apes the prefrontal cortex is indeed larger than predicted on the basis of the primary visual cortex. For the Brodmann dataset, the human value is 9.64 times larger than predicted on the basis of data for the monkeys and gibbon $(\mathrm{p}<0.07)$. The chimpanzee value is 2.38 times larger than predicted on the same basis. For the Smaers dataset, the human value is 8.10 times larger than predicted on the basis of the data for the monkeys and gibbon $(\mathrm{p}<0.05)$. The chimpanzee value is 3.38 times larger than predicted, and the values are similar for the other great apes $(\mathrm{p}<$ 0.05).

We therefore propose that in relation to the prefrontal cortex there are three grades. The first constitutes the monkeys, and the gibbon does not appear to be distinct from this, the second constitutes the great apes and the third is represented by modern humans.

\section{Is the Human Prefrontal Cortex Especially Enlarged}

Relative to Other Association Areas?

Though the previous section has established that the prefrontal cortex is especially enlarged in the human brain, it is clear that it is not the only area that is enlarged. For the Smaers dataset the motor areas are significantly enlarged. It is likely that, if we had separate measures for the premotor areas and primary motor cortex, the difference would lie in the premotor association areas.

For both datasets the human nonfrontal minus striate cortex is also significantly enlarged. It is unfortunate that our measure of nonfrontal minus striate cortex includes both the primary somatosensory and auditory cortex. However, we have no separate measure of these, and are therefore unable to subtract them so as to reach a purer measure of posterior association cortex.

For both the Brodmann and Smaers datasets the human prefrontal cortex is significantly enlarged relative to the motor areas. For the Smaers dataset it is also significantly enlarged relative to the nonfrontal minus striate cortex. For the Brodmann dataset the results for the latter comparison do not quite reach significance. However, the values for the residuals in the two datasets are similar, though not identical.

Is the Prefrontal Cortex Especially

Enlarged in the Human Brain?
We suggest that one possible reason for any differences in the results for the two datasets concerns the slopes. The slope is isometric in the Smaers dataset (table 1) and hypermetric in the Brodmann dataset (table 1). If the slope is hypermetric this will decrease the size of the residual for the human brain. However, we have shown that the slope for the Brodmann dataset is no longer hypermetric if the marmoset is excluded. That the marmoset should be excluded is suggested by studies of its prefrontal cortex. Unlike the squirrel monkey [Lewis et al., 1986], for example, it does not have a distinct area 46 or 9 [Roberts et al., 2007]. In this respect it is similar to a prosimian, such as a bushbaby [Preuss and Goldman-Rakic, 1991a, b].

There is another reason for relying on the results for the Smaers dataset. It has the advantage that it provides values for more monkeys and also for each of the 4 great apes. We mentioned in the introduction that it is not clear whether the proper reference group is anthropoids or the great apes in particular. For this reason table 2 shows the ratio of observed to predicted values both compared with the data for monkeys and the gibbon alone, and for the non-human anthropoids including the 4 great apes. The human prefrontal cortex is significantly enlarged on both analyses.

\section{Comparison of our Results with Those in the Literature}

We see four possible reasons why our conclusion differs from that of some other analyses in the literature. The first is that we have used measures of the prefrontal cortex whereas others have used measures of the frontal cortex as a whole [Semendeferi et al., 2002; Barton and Venditti, 2013]. It is clear from the work of Semendeferi and Damasio [2000] that the frontal, temporal and combined parietal plus occipital lobe form the same proportions of the cortex in the human as in the chimpanzee brain. However, if the chimpanzee brain is morphed so as to align with the human brain [Avants et al., 2006] it becomes clear that there has been an expansion of the association areas within each of these sectors in the human brain. Controlling for brain volume, there has also been a marked expansion in the anterior insula of the human brain when compared to the chimpanzee brain [Bauernfeind et al., 2013]. These conclusions are supported by our own finding that the prefrontal cortex is greater in the human brain than predicted for an anthropoid matched for the size of the premotor plus motor cortex.

A second possible reason why our conclusion differs is that we have been careful to ensure that our measure is of the prefrontal cortex alone. We have done this either by using Brodmann's cytoarchitectonic data or by ensur- 
ing that the volume measured is anterior enough to avoid the entire premotor cortex. Schoenemann et al. [2005] also took an anterior cut, but the level of their cut leaves open the possibility that the measure of prefrontal cortex in the great apes is an overestimate [Sherwood et al., 2005] because it includes some of the dysgranular frontal cortex [Passingham, 2008]. In the human brain, area 6 extends superiorly beyond the precentral sulcus [Geyer, 2004], but the dysgranular frontal cortex extends much further in the chimpanzee brain [Bailey et al., 1950].

A third possible reason for the difference concerns the comparison group. Semendeferi et al. [2002] used great apes as the comparison group. For the Smaers dataset, we were able to use regressions through the data for anthropoids, including the great apes, to show that the human prefrontal cortex is especially enlarged, but also to use data for monkeys and the gibbon to show that that the prefrontal cortex is also especially enlarged in great apes.

A final reason concerns the slopes of the regression lines. For the Smaers dataset the slopes for the regression lines for monkeys and the gibbons are isometric. However, in the study by Semendeferi et al. [2001] of the frontopolar area 10, the slope is strongly hypermetric. In that case the line is fitted through the values for the gibbon and 4 great apes, and is highly influenced by the value for the gibbon as an outlier. Our results are based on a broader comparative sample. They suggest that the gibbon can be considered as an outlier as an ape because it scales with monkeys, not great apes, and therefore constitutes part of a different grade.

\section{Remapping Factors}

It will be clear that, though the prefrontal cortex is enlarged in great apes and humans, so are other areas of the cortex. However, the analysis of the data for the human brain shows that this is not true of the primary sensory areas. As mentioned in the introduction, the striate cortex [Passingham, 1973] and koniocortex as a whole [Passingham, 1975] are as predicted for a primate our body size. It is the nonprimary areas that have expanded. The same point is made by the analysis of the distribution of myelin in the cortex [Glasser et al., 2013]. The higher-order association areas are less densely myelinated, and it is these that are especially expanded in the human compared with the chimpanzee brain.

There must be a consequence for processing capacity. Consider, for example, the analysis of visual information. The striate cortex projects via V2 and V4 to the inferotemporal cortex [van Essen, 2005]. Both the human brain and the chimpanzee brain have a middle as well as an inferior temporal gyrus [Passingham, 2008], and yet the remapping factor is roughly three and a half times bigger in the human brain (table 3 ).

The striate cortex also projects via V3, MT/V5 and V6 to the parietal cortex [van Essen, 2005]. Unfortunately, we do not have a direct measure of the size of the parietal association cortex. The measure we have used of the nonfrontal minus striate cortex includes not only the prestriate cortex, but also SI and SII. As explained in the Methods section we have calculated a 'parietal estimate', and this includes the prestriate cortex. The remapping factor for this estimate is roughly two and a half times that in the human compared with the chimpanzee brain (table 3 ).

The prefrontal cortex does not receive direct inputs from the striate cortex. Instead it derives its cortical inputs from the parietal and temporal cortex [Petrides and Pandya, 1999, 2002]. The prefrontal cortex acts to integrate information from these areas so as to establish the current context [Passingham and Wise, 2012]. The fact that the scaling is isometric in monkeys suggests that the amount of prefrontal tissue that is needed for this purpose is a function of the amount of information that it receives from the parietal and temporal cortex.

However, there is a step change in the remapping factor in the chimpanzee brain, and then again in the human brain (table 3). The factor is around sixty percent greater between the macaque and the chimpanzee, and again around sixty percent between the chimpanzee and the human. The implication is that, compared with a chimpanzee or macaque monkey brain, the human prefrontal cortex is especially equipped to integrate information about the context. We suggest in a later section that this includes the integration of information about different relations.

The prefrontal cortex specifies the spatial and object goals of action [Yamagata et al., 2012]. These can include a series of goals as in planning [Mushiake et al., 2006] or performing [Shima et al., 2007] sequences of action. These in turn are achieved by the operation of the premotor and motor areas to which the prefrontal cortex projects [Wang et al., 2002; Takahara et al., 2012]. The prefrontal cortex should therefore also be considered in relation to its outputs. The remapping factor from prefrontal to frontal motor areas is nearly four times as large in the human as in the chimpanzee brain (table 3 ). The implication is that, compared to the chimpanzee or macaque monkey brain, the human brain is especially equipped to generate a series of goals. 


\section{Limitations of the Study}

It is a limitation of the study that the remapping factors have been worked out in terms of tissue rather than cell numbers. For remapping, what matters is the number of cells that are available in higher areas for processing the inputs from earlier areas. While we have data on average cell density for a range of primates including humans [Herculano-Houzel et al., 2007], we need data on the number of cells in each functional area. It is clear from the work of Ribeiro et al. [2013] that in the human brain the density of cells decreases as one moves anteriorly in the brain, being at its lowest in the anterior sections. It is also clear that in the human brain the cell density in the frontal polar cortex is particularly sparse [Semendeferi et al., 2010; Spocter et al., 2012]. Thus, it could be that if, for example, cell numbers were available for the prefrontal and temporal cortex, it might turn out that the human remapping factor does not stand out in terms of cells, though it does in terms of tissue.

Whether the calculation is in terms of tissue or cells, it provides no information about the degree to which information from different inputs can be integrated onto single cells. Yet Passingham and Wise [2012] have stressed the importance of the prefrontal cortex in integrating inputs from posterior areas. The inputs terminate on the dendritic spines, and measurements have been taken of the number of these on layer III pyramidal cells in the human and chimpanzee brain [Bianchi et al., 2013]. However, it is important to calculate the remapping factors for these between prefrontal and other areas because this corrects for differences in cell size that are due to differences in overall brain size. For the number of spines in area 10 compared with visual area 18 , the figures for the remapping factors are on average 1.31 in the human brain and 2.36 in the chimpanzee brain, though it is clear from the statistical analyses in the paper that the differences between species are not significant. The figures for the remapping factors for area 10 versus motor cortex area 4 are 1.64 in the human brain and 2.42 in the chimpanzee brain. These figures do not suggest greater prefrontal integration onto pyramidal cells in the human brain.

\section{Conclusions}

Our allometric analyses suggest that the prefrontal cortex is indeed enlarged in the human compared with the chimpanzee brain. However, it also appears to be especially enlarged in the great ape brain compared with the brains of monkeys. We suggest that modern humans and great apes form two distinct grades, and that they both differ from monkeys.

Our analysis of the remapping factors suggests that these differences have consequences for processing capacity. However, further work is needed to check whether the differences hold up when the number and size of the cells is taken into account.

\section{Acknowledgements}

We thank Steve Wise and Chet Sherwood for detailed comments on the manuscript.

\section{References}

Avants BB, Schoenemann PT, Gee JC (2006): Lagrangian frame diffeomorphic image registration: morphometric comparison of human and chimpanzee cortex. Med Image Anal 10: 397-412.

Bailey P, von Bonin G (1951): The Isocortex of Man. Urbana, University of Illinois Press.

- Bailey P, von Bonin G, McCullogh WS (1950): The Isocortex of the Chimpanzee. Urbana, University of Illinois Press.

Barton RA, Harvey PH (2000): Mosaic evolution of brain structure in mammals. Nature 405: 1055-1058.

- Barton RA, Venditti C (2013): Human frontal lobes are not relatively large. Proc Natl Acad Sci USA 110:9001-9006.

- Bauernfeind AL, de Sousa AA, Avasthi T, Dobson SD, Raghanti MA, Lewandowski AH, Zilles K, Semendeferi K, Allman JM, Craig AD, Hof
PR, Sherwood CC (2013): A volumetric comparison of the insular cortex and its subregions in primates. J Hum Evol 64:263-279.

-Bianchi S, Stimpson CD, Bauernfeind AL, Schapiro SJ, Baze WB, McArthur MJ, Bronson E, Hopkins WD, Semendeferi K, Jacobs B, Hof PR, Sherwood CC (2013): Dendritic morphology of pyramidal neurons in the chimpanzee neocortex: regional specializations and comparison to humans. Cereb Cortex 23: 2429-2436.

Brodmann K (1913): Neue Forchungsergebnisse der Grosshirnrindeanatomische mit besonderer Berucksichtung anthropologischer Fragen. Gesselch Deuts Naturf Artze 85:200-240.

Bush EC, Allman JM (2004): Three-dimensional structure and evolution of primate primary visual cortex. Anat Rec A Discov Mol Cell Evol Biol 281:1088-1094.
Cowey A, Ellis CM (1969): The cortical representation of the retina in squirrel and rhesus monkeys and its relation to visual acuity. Exp Neurol 24:374-385.

de Sousa AA, Sherwood CC, Mohlberg H, Amunts K, Schleicher A, MacLeod CE, Hof PR, Frahm H, Zilles K (2010): Hominoid visual brain structure volumes and the position of the lunate sulcus. J Hum Evol 58:281-292.

Deacon RM (1997): The Symbolic Species. London, Allen Lane.

Desimone R, Schein SJ (1987): Visual properties of neurons in area $\mathrm{V} 4$ of the macaque: sensitivity to stimulus form. J Neurophysiol 57:835-868.

Elston GN (2007): Specialization of the neocortical pyramidal cell during primate evolution; in Kaas J, Preuss TM (eds): Evolution of Nervous Systems: A Comprehensive Reference, vol. 4. New York, Elsevier, pp 191-242.
Is the Prefrontal Cortex Especially

Enlarged in the Human Brain?
Brain Behav Evol 2014;84:156-166 DOI: $10.1159 / 000365183$ 
-Frahm HD, Stephan H, Baron G (1984): Comparison of brain structure volumes in insectivora and primates. V. Area striata. J Hirnforsch 25: 537-557.

Geyer S (2004): The microstructural border between the motor and the cognitive domain in the human cerebral cortex. Adv Anat Embryol Cell Biol 174:1-89.

- Glasser MF, Goyal MS, Preuss TM, Raichle ME, van Essen DC (2014): Trends and properties of human cerebral cortex: correlations with cortical myelin content. Neuroimage 93:165175.

-Herculano-Houzel S, Collins CE, Wong P, Kaas JH (2007): Cellular scaling rules for primate brains. Proc Natl Acad Sci USA 104:35623567.

Holloway RL (2002): Brief communication: how much larger is the relative volume of area 10 of the prefrontal cortex in humans? Am J Phys Anthropol 118:399-401.

Kaas JH (1997): Topographic maps are fundamental to sensory processing. Brain Res Bull 44:107-112.

Kelly C, Price TD (2004): Comparative methods based on species mean values. Math Biosci 187:135-154.

Kumar S, Filipski A, Swarna V, Walker A, Hedges SB (2005): Placing confidence limits on the molecular age of the human-chimpanzee divergence. Proc Natl Acad Sci USA 102:1884218847.

Lewis DA, Campbell MJ, Morrison JH (1986): An immunohistochemical characterization of somatostatin-28 and somatostatin-281-12 in monkey prefrontal cortex. J Comp Neurol 248:1-18.

Mackey S, Petrides M (2010): Quantitative demonstration of comparable architectonic areas within the ventromedial and lateral orbital frontal cortex in the human and the macaque monkey brains. Eur J Neurosci 32:1940-1950.

-Mushiake H, Saito N, Sakamoto K, Itoyama Y, Tanji J (2006): Activity in the lateral prefrontal cortex reflects multiple steps of future events in action plans. Neuron 50:631-641.

Orme D, Frecketon R, Thomas G, Petzolt T, Fritz S, Isaac N, Pearse W (2012): Caper: comparative analyses of phylogenetics and evolution in R. http://CRAN.R-project.org/ package $=$ caper

- Passingham RE (1973): Anatomical differences between the neocortex of man and other primates. Brain Behav Evol 7:337-359.

Passingham RE (1975): Changes in the size and organization of the brain in man and his ancestors. Brain Beh Evol 11:73-90.

Passingham RE (1981): Primate specialization in brain and intelligence. Symp Zool Soc Lond 46:361-368.

- Passingham RE (2008): What Is Special About the Human Brain. Oxford, Oxford University Press.

Passingham RE, Wise SP (2012): The Neurobiology of Prefrontal Cortex. Oxford, Oxford University Press.
Petrides M, Pandya DN (1999): Dorsolateral prefrontal cortex: comparative cytoarchitectonic analysis in the human and the macaque brain and corticocortical connection patterns. Eur J Neurosci 11:1011-1036.

Petrides M, Pandya DN (2002): Comparative cytoarchitectonic analysis of the human and macaque ventrolateral prefrontal cortex and corticocortical connection pattern in the monkey. Eur J Neurosci 16:291-310.

Preuss TM, Goldman-Rakic PS (1991a): Ipsilateral cortical connections of granular frontal cortex in the strepsirhine primate Galago, with comparative comments on anthropoid primates. J Comp Neurol 310:507-549.

Preuss TM, Goldman-Rakic PS (1991b): Myeloand cytoarchitecture of the granular frontal cortex and surrounding regions in the strepsirhine primate Galago and the anthropoid primate Macaca. J Comp Neurol 310:429474.

Rao C, Toutenburg H (1999): Linear Models: Least Squares and Alternatives. New York, Springer.

Ribeiro PF, Ventura-Antunes L, Gabi M, Mota B, Grinberg LT, Farfel JM, Ferretti-Rebustini RE, Leite RE, Filho WJ, Herculano-Houzel S (2013): The human cerebral cortex is neither one nor many: neuronal distribution reveals two quantitatively different zones in the gray matter, three in the white matter, and explains local variations in cortical folding. Front Neuroanat 7:28.

Rilling JK, Seligman RA (2002): A quantitative morphometric comparative analysis of the primate temporal lobe. J Hum Evol 42:505533.

Roberts AC, Tomic DL, Parkinson CH, Roeling TA, Cutter DJ, Robbins TW, Everitt BJ (2007): Forebrain connectivity of the prefrontal cortex in the marmoset monkey (Callithrix jacchus): an anterograde and retrograde tracttracing study. J Comp Neurol 502:86-112.

Rohlf FJ (2001): Comparative methods for the analysis of continuous variables: geometric interpretations. Evolution 55:2143-2160.

Schoenemann PT, Sheehan MJ, Glotzer LD (2005): Prefrontal white matter volume is disproportionately larger in humans than in other primates. Nat Neurosci 8:242-252.

- Semendeferi K, Armstrong E, Schleicher A, Zilles K, van Hoesen GW (2001): Prefrontal cortex in humans and apes: a comparative study of area 10. Am J Phys Anthropol 114:224-241.

-Semendeferi K, Damasio H (2000): The brain and its main anatomical subdivisions in living hominoids using magnetic resonance imaging. J Hum Evol 38:317-332.

-Semendeferi K, Lu A, Schenker N, Damasio H (2002): Humans and great apes share a large frontal cortex. Nat Neurosci 5:272-276.

Semendeferi K, Teffer K, Buxhoeveden DP, Park MS, Bludau S, Amunts K, Travis K, Buckwalter J (2011): Spatial organization of neurons in the frontal pole sets humans apart from great apes. Cereb Cortex 21:1485-1497.
Shariff GA (1953): Cell counts in the primate cerebral cortex. J Comp Neurol 98:381-400.

-Sherwood CC, Holloway RL, Semendeferi K, Hof PR (2005): Is prefrontal white matter enlargement a human evolutionary specialization? Nat Neurosci 8:537-538.

Sherwood CC, Smaers JB (2013): What's the fuss over human frontal lobe evolution? Trends Cogn Sci 17:432-433.

- Shima K, Isoda M, Mushiake H, Tanji J (2007): Categorization of behavioural sequences in the prefrontal cortex. Nature 445:315-318.

Smaers JB (2013): How humans stand out in frontal lobe scaling. PNAS 110:E3682.

-Smaers JB, Mulvaney PI, Soligo C, Zilles K, Amunts K (2012): Sexual dimorphism and laterality in the evolution of the primate prefrontal cortex. Brain Behav Evol 79:205-212.

-Smaers JB, Schleicher A, Zilles K, Vinicius L (2010): Frontal white matter volume is associated with brain enlargement and higher structural connectivity in anthropoid primates. PLoS One 5:e9123.

-Smaers JB, Steele J, Case CR, Amunts K (2013): Laterality and the evolution of the prefrontocerebellar system in anthropoids. Ann NY Acad Sci 1288:59-69.

Smaers JB, Steele J, Case CR, Cowper A, Amunts K, Zilles K (2011): Primate prefrontal cortex evolution: human brains are the extreme of a lateralized ape trend. Brain Behav Evol 77:6778.

- Spocter MA, Hopkins WD, Barks SK, Bianchi S, Hehmeyer AE, Anderson SM, Stimpson CD, Fobbs AJ, Hof PR, Sherwood CC (2012): Neuropil distribution in the cerebral cortex differs between humans and chimpanzees. J Comp Neurol 520:2917-2929.

Takahara D, Inoue K, Hirata Y, Miyachi S, Nambu A, Takada M, Hoshi E (2012): Multisynaptic projections from the ventrolateral prefrontal cortex to the dorsal premotor cortex in macaques - anatomical substrate for conditional visuomotor behavior. Eur J Neurosci 36:3365-3375.

Tanaka K (1993): Neuronal mechanisms of object recognition. Science 262:685-688.

van Essen DC (2005): Corticocortical and thalamocortical information flow in the primate visual system. Prog Brain Res 149:173-185.

Wang Y, Shima K, Osoda M, Sawamura H, Tanji J (2002): Spatial distribution and density of prefrontal cortical cells projecting to three sectors of the premotor cortex. Neuroreport 13:1341-1344.

-Yamagata T, Nakayama Y, Tanji J, Hoshi E (2012): Distinct information representation and processing for goal-directed behavior in the dorsolateral and ventrolateral prefrontal cortex and the dorsal premotor cortex. J Neurosci 32:12934-12949.

Zilles K, Amunts K, Smaers JB (2011): Three brain collections for comparative neuroanatomy and neuroimaging. Ann NY Acad Sci 1225(suppl 1):E94-E104. 


\section{Erratum}

In the article by Passingham RE and Smaers JB, entitled 'Is the prefrontal cortex especially enlarged in the human brain? Allometric relations and remapping factors' [Brain Behav Evol 2014;84:156-166, DOI: 10.1159/000365183], Passingham and Smaers used the confidence interval on the intercept as the criterion for identifying an outlier. They wrongly stated that this was the procedure adopted by Barton and Harvey [2000] and Barton and Venditti [2013]. In fact Barton and Venditti [2013] used the prediction interval for the individual value as this accounts for uncertainty in both the intercept and the slope [R.A. Barton, pers. commun.]. When the data used by Passingham and Smaers are analysed using the confidence intervals accounting for the uncertainty of the intercept and the slope, the results hold. 